\title{
A New Cosmological Paradigm: the Cosmological Constant and Dark Matter
}

\author{
Lawrence M. Krauss ${ }^{1}$ \\ Departments of Physics and Astronomy \\ Case Western Reserve University, Cleveland, OH 44106-7079
}

\begin{abstract}
The Standard Cosmological Model of the 1980's is no more. I describe the definitive evidence that the density of matter is insufficient to result in a flat universe, as well as the mounting evidence that the cosmological constant is not zero. I finally discuss the implications of these results for particle physics and direct searches for non-baryonic dark matter, and demonstrate that the new news is good news.
\end{abstract}

\section{INTRODUCTION}

One of the great developments of the 1980's was the creation of a Standard Model of Cosmology based on ideas arising from Particle Theory. This model involved the following trilogy of ideas:

$$
\begin{gathered}
\Omega \equiv 1 \\
\Lambda \equiv 0 \\
\Omega_{\text {matter }} \approx \Omega_{\mathrm{CDM}}^{\text {axion }} \underset{\text { WIMP }}{ } \geq 0.9
\end{gathered}
$$

A decade later observational cosmology has made tremendous strides, and we now know that at least two of these fundamental notions must be incorrect. Either

1) Material based on Invited Lectures, PASCOS 98, Boston MA, March 98; Tropical Workshop on Particle Physics and Cosmology, San Juan, Puerto Rico, April 98; WEIN98, Santa Fe, NM, June 98. To appear in these proceedings. Research supported in part by the DOE. email:krauss@theory1.phys.cwru.edu 


$$
\begin{gathered}
\Omega \neq 1 \\
\Lambda \equiv 0 \\
\Omega_{\text {matter }} \approx \Omega_{\mathrm{CDM}}^{\text {axion }} \underset{\text { WIMP }}{ } \approx 0.1-0.3
\end{gathered}
$$

or

$$
\begin{gathered}
\Omega \equiv 1 \\
\Lambda \neq 0 \\
\Omega_{\text {matter }} \approx \Omega_{\mathrm{CDM}_{\text {axion }}^{\mathrm{WIMP}}} \approx 0.1-0.3
\end{gathered}
$$

In either case the implications for both cosmology and particle physics are profound. In the first place,

Either: $\Omega \neq 1$ or $\Lambda \neq 0$

Whichever is true, this implies we don't understand something very fundamental about the microphysics of the Universe - a very exciting prospect! If $\Omega \neq 1$ then the canonical prediction of inflation is incorrect, and we have to understand how inflation, or another theory, might address the fine tuning required to solve the flatness problem without actually resulting in a flat universe today. If $\Lambda \neq 0$ then the situation is in a sense even more exciting, as there is no theory of the cosmological constant at the present time, and the supposition that this quantity is indeed zero rests primarily on a priori theoretical prejudice at this point. (I here include in the term "cosmological constant" those models which involve a very slowly varying scalar field, which in effect mimics a cosmological constant over long time intervals.)

At the same time, we have:

$$
\Omega_{\text {matter }} \approx \Omega_{\mathrm{CDM}_{\text {axion }}^{\mathrm{WIMP}}} \approx 0.1-0.3
$$

This also has dramatic implications, not only for our understanding of the role dark matter plays in the formation of large scale structure, but also for our propsects for direct detection of non baryonic dark matter. Contrary to one's naive expectations however, the implications are quite positive. Dark matter may not contribute $90 \%$ of the mass of the Universe, as previously envisaged, but it still appears to outweigh baryonic matter. Moreover, as I will demonstrate, in all cases these results suggest that the interaction strength of dark matter with normal matter will be INCREASED, and thus in principle direct detection should be easier 
than it would otherwise be. As long as the dark matter contribution to the fraction of the closure density is larger than 0.1, so that it can account for essentially the entire inferred dark matter content of galactic halos in general, and our galactic halo in particular, the increase in interaction cross section is not counterbalanced by a decrease in the dark matter flux on earth, so that the net event rate in detectors should be larger than would be the case if $\Omega_{\mathrm{CDM}} \approx 0.9$.

\section{THE CASE FOR A COSMOLOGICAL CONSTANT}

Over the past 5 years a variety of indirect observables, involving the three fundamental independent observables in cosmology, the expansion rate, the matter content, and large scale structure, have all suggested that either the universe is open, or the cosmological constant is not zero [1-3]. In the past year, the indirect evidence has been strengthened by new large scale structure measurements, and for the first time, striking new direct measurements suggest that the Hubble expansion is accelerating. I first review the older, indirect evidence, and then describe the most recent results.

\section{A The Age Problem}

The Hubble constant, by a very simple argument, gives an upper limit on the age of a matter dominated universe. Matter causes a deceleration of the universal expansion over time. Thus, at earlier times the universe would have been expanding faster than it is at the present time. One can, in turn, therefore derive an upper limit on the age of the universe by considering the fact that all galaxies were once located together, and using the relation for a constant velocity to determine the length of time a galaxy at a given distance, moving away at a constant velocity took to get there, i.e. $d=v t \rightarrow t=d / v=H_{0}^{-1}$, where the definition of the Hubble constant, $H_{0}$ has been used. In fact, of course, this upper limit on the age of the Universe is an overestimate of its age, and with a given cosmological model one can derive a specific relation between the Hubble constant today and the age of the universe. One has:

Flat matter dominated $\quad t=(2 / 3) H_{0}^{-1}=9.7 \mathrm{Gyr}\left(65 / H_{0}\right)$

$$
\text { Open }(\Omega>0.2) \quad t<.85 H_{0}^{-1}=12.5 \mathrm{Gyr}\left(65 / H_{0}\right)
$$

Flat $\left(\Omega_{\Lambda}<0.8\right) \quad t<1.08 H_{0}^{-1}=16 \mathrm{Gyr}\left(65 / H_{0}\right)$

Thus, if one could definitively demonstrate that the Universe were older than either of the first two relations allowed, given the allowed range of $H_{0}$, one would have strong evidence that $\Lambda \neq 0$, since a non-zero cosmological constant would allow a universal acceleration, and hence allows an older universe for a fixed Hubble Constant. 
While precisely such a situation seemed to prevail as recently as 1996 [4], more recent estimates for the age of globular clusters have suggested that the age of our galaxy is younger than previously estimated. At the same time, estimates of the Hubble constant are now somewhat lower than previously claimed, so that a range of $65-75 \mathrm{kms}^{-1} \mathrm{Mpc}^{-1}$ is now preferred [5]. Nevertheless, the new quoted $95 \%$ lower limit, of approximately 9.8 Gyr with a best estimate of the age of 11.5 Gyr [6], strongly disfavors a flat universe, even if it remains compatible with an open universe.

\section{B The Baryon Problem}

Big Bang Nucleosynthesis (BBN) has for some time provided an upper limit on the total density of baryonic matter in the univere $[7,8]$ :

$$
\Omega_{B} h^{2} \leq .026
$$

Most recently, claimed observations of the deuterium fraction in primordial hydrogen clouds illuminated by the light of distant quasars [9] suggest that the actual baryon abundance is near the upper limit of this range. While this puts pressure on BBN analyses, more germaine for this discussion is the fact that when combined with direct observations of the baryon fraction on large scales today, it effectively rules out the possibility of a flat universe.

X-Ray Observations of Clusters of Galaxies, the largest bound structures known in the Universe suggest that the dominant baryonic material in these systems exists in the form of hot X-Ray emitting gas. Assuming this material is in hydrostatic equilibrium with the gravitational potential of these systems one can, by observing both the X-Ray Luminosity and temperature as a function of radius, perform an inversion which gives an estimate of this potential, and hence the total mass, $M_{T}$, of these. At the same time, direct observations of the luminosity yield an estimate for the total baryonic mass in hot gas, and hence the total baryonic mass $M_{B}$. Thus, one derives the ratio $R=M_{B} / M_{T}$ for these sytems. Now, as these systems are the largest bound objects known, it is reasonable to assume that they are good probes of the distribution of all gravitating matter on large scales. Thus, the ratio $\mathrm{R}$ is expected to be not just the ratio of baryon to total mass of clusters, but the ratio of baryonic to total mass in the Universe. Thus, if the Universe is flat, so that the density corresponding to $M_{T}$ yields $\Omega=1$ then one has precisely the relation $R=\Omega_{B}$. Therein lies the problem. Observations, combined with theoretical models of clusters yield the constraint $[10]$

$$
R>.043 h^{-3 / 2}
$$

If $R=\Omega_{B}$ then clearly this equation is inconsistent with the BBN bound.

This problem can be simply resolved however, if $\Omega_{M_{T}}<1$ so that the ratio $\mathrm{R}$ is in fact larger than $\Omega_{B}$. There are then two possibilities. Either $\Omega_{M_{T}}=\Omega<1$, or $\Omega_{M_{T}}+\Omega_{\Lambda}=1$. 


\section{Large Scale Structure}

The growth of structure in the Universe, if gravity is responsible for such growth, provides an excellent probe of the universal mass density, based largely on issues associated with causality alone. The basic idea is the following: If primordial density fluctuations have no preferred scale, then one can express their Fourier transform as a simple power of the wavenumber $k$. At the same time, if this power is much greater than unity, density fluctuations will blow up for large wavenumber, or small wavelength, and too many primordial black holes will be created. If the power is much less than unity, then fluctuations on large scales (small wavenumbers) will be inconsistent with the observed isotropy of the Cosmic Microwave Background radiation. Thus, we expect the exponent, $n$ to be near one, and inflationary models happen to predict precisely this behavior.

The primordial power spectrum, however, is not what we observe today, as density fluctuations can be affected by causal microphysical processes once the scale of these fluctuations is inside the horizon scale - the distance over which light can have travelled between $t=0$ and the time in question. One can show that in an expanding universe, as long as the dominant form of energy resides in radiation, gravity is ineffective at causing the growth of density fluctuations. In fact, such primordial fluctuations in baryons will be damped out due to their coupling to the radiation gas. Once the universe becomes matter dominated, however, primordial fluctuations on scales smaller than the horizon size can begin to grow.

\section{Fluctuation Spectrum}
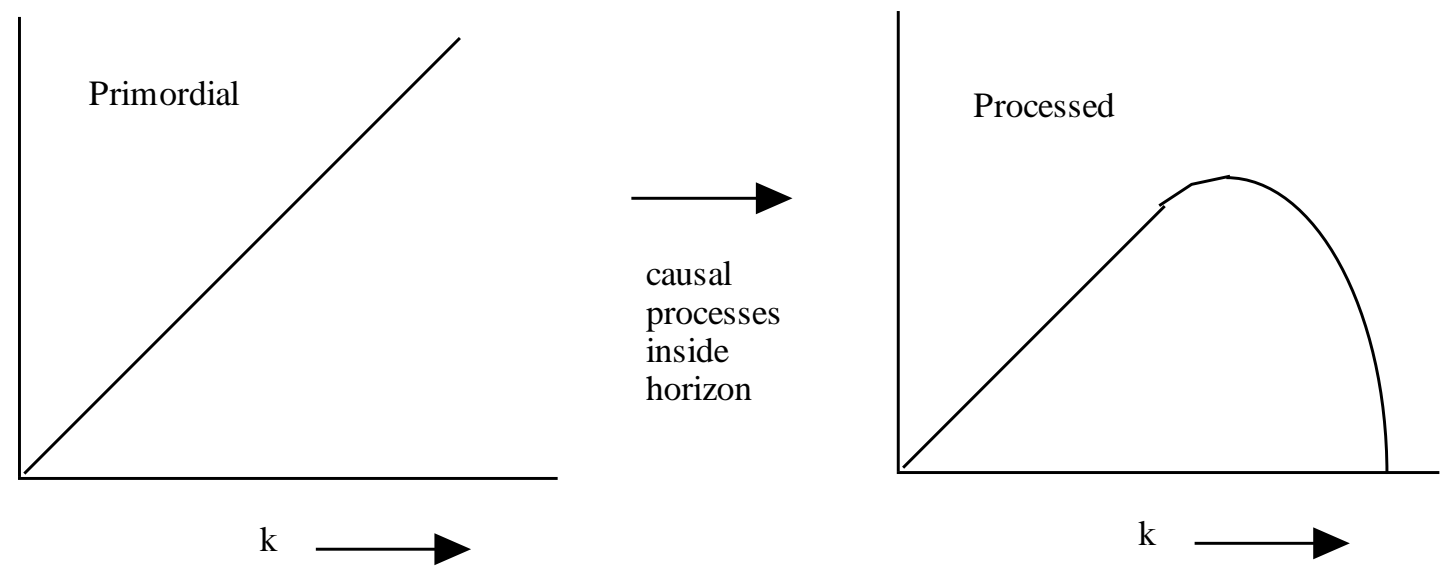

These arguments suggest that an initial power law spectrum of fluctuations will "turn over" as shown in Figure 1 above for large wavenumbers which entered inside the causal horizon during the early period of radiation domination in the Universe. By exploring the nature of the clustering of galaxies today over different scales, including measurements of the two point correlation function of galaxies, the angular correlation of galaxies across the sky on different scales, etc, one can hope to 
probe the location of this turn-around, and from that probe the time, and thus the scale which first entered the horizon when the universe became matter dominated. Clearly this time will depend upon the ratio of matter to radiation in the Universe today (if this ratio is increased, then matter, whose density decreases at a slower rate than radiation as the universe expands, will begin to dominate the expansion at an earlier time, and vice versa. In turn, knowing this ratio today gives us a handle on $\Omega_{\text {matter }}$. A recent compilation of large scale galaxy clustering data $[11,12]$ restricts this quantity to be in the range:

$$
0.25 \leq \Omega_{\text {matter }} h \leq 0.35
$$

Since $h$ appears to lie in the range $0.65-0.75$, this suggests $\Omega_{\text {matter }}<1$. Note, however that this argument does not restrict the component of $\Omega$ which might reside in a cosmological constant today, since this energy density is fixed, so that even if it dominates today, it was irrelevant compared to the energy density of matter and radiation at early times.

By combining these three independent sets of constraints, one can, for either an open universe, or a flat universe with a cosmological constant, constrain the parameter space of $h$ versus $\Omega_{\text {matter }}[1,3]$. These constraints are shown in the figures below [3], which clearly indicate that a flat matter dominated universe appears to be inconsistent with observations.

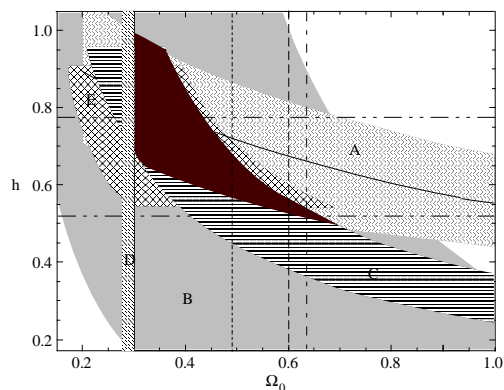

FIGURE 1. Constraints on $h$ vs $\Omega_{\text {mat }}$ for a flat universe. The constraints discussed in the text (A, B, and $\mathrm{C}$ ). The other constraints are discussed in (Krauss 98).

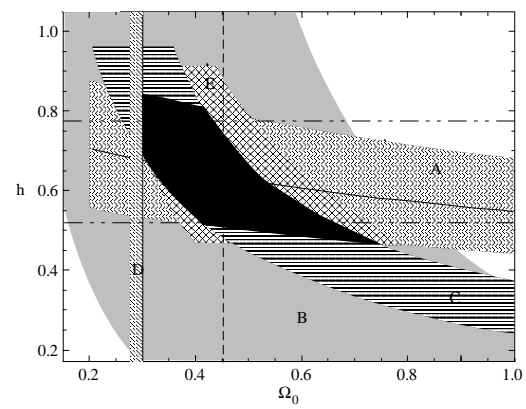

FIGURE 2. Constraints on $h$ vs $\Omega_{\text {matter }}$ for an open universe. Constraints are as described in previous figure.

\section{Recent Observations: Galaxy Clustering, the CMB and Supernova Standard Candles}

The situation described above has been recognized for over two years. Within the last year or so, however, new observations have made the case for a cosmological constant even stronger. I briefly describe these observations below. 
(1) Cluster Evolution: In a Universe in which there is not a precisely critical density of matter, small density fluctuations on large scales cease to grow due to gravity once either the total density begins to deviate significantly from that associated with a flat universe, or when the density in a cosmological term begins to dominate over the density of matter. Thus, if the universe is not flat today, or if the cosmological constant dominates, then structure has ceased to continue to grow on large scales. If, however, the universe is flat and matter dominated, structures on every larger scales are continuing to form by gravitational collapse. This suggests that if one examines out to high redshifts one should see significantly fewer rich clusters of galaxies then one sees today. The difference is significant. As Neta Bahcall and colleagues have recently pointed out [13], the probability of finding a rich cluster at a redshift of 0.7 is perhaps 100 times smaller for a flat matter dominated universe than for a universe in which the growth of large structures stopped some time ago. A single large cluster observed at such high redshift can then provide, largely independent of detailed modelling, damning evidence against a flat matter dominated universe. By cataloguing such clusters out to redshifts of this order, these authors have recently claimed to present definitive evidence ruling out a flat, cold dark matter dominated universe. Both open, or flat cosmological constant dominated universes are consistent with this data.

(2) Type 1a Supernova at High Redshift: As anyone who has glanced at a paper in the past six months knows, two groups have recently and hopefully independently claimed to measure the relation between redshift and distance out to redshifts in excess of 0.5 , and in so doing have been able to probe for cosmic deceleration or acceleration. The probes used have been Type 1a Supernovae. These have been claimed to be superb Standard Candles for two reasons: (i) Type 1a supernovae occur when a white dwarf, through accretion, passes the Chandrsekhar limit, and undergoes a detonation explosion. The physics of this process should not depend significantly on the evolutionary status of the galaxy in which the star is housed. (ii) Detailed studies of the luminosity profile of such supernovae suggest a strong relation between the width of the light curve, and the absolute luminosity of the supernova. This allows one, in principle, to accurately determine this absolute luminosity. Based on these features both groups have now claimed to report definitive evidence for a non-zero cosmological constant. Moreover, they claim, at the $99 \%$ confidence level, to be able to rule out both a flat, and an open universe with zero cosmological constant [14,15]. Even more remarkably, the favored region, for a flat universe, is precisely in the range favored by the other constraints in the figures shown above (which I remind you were drawn before these results appeared), namely $\Omega_{\text {mat }} \approx 0.3-0.4, \Omega_{\Lambda} \approx 0.6-0.7$. It remains to be seen if further data taken at high redshift confirm these results, and more importantly confirm the assumption that evolution is negligible for such supernovae.

(3) CMB preliminary studies: If one decomposes the observed CMB anistropies on the sky into multipoles on the sky, it is well known that CDM cosmologies predict a rise in the power spectrum as a function of multipole approaching a large peak, followed by smaller peaks. The position, in multipole space, of this first peak 
is a probe of the geometry of the universe, as it is related to the angular size of the horizon at the last scattering surface, as seen today. Very preliminary results from terrestrial observations of high multipole anisotropies in the CMB tend to confirm the existence of such a peak, and moreover the position of the peak appears to favor a flat, versus an open universe. If this is the case, then the existence of a non-zero cosmological constant, in light of all the other direct and indirect evidence, seems assured.

It is almost unnerving that all existing cosmological data appears to point in the same direction - towards a non-zero cosmological constant. As someone who has been promoting the idea that the cosmological constant might be non-zero for some time, I frankly found myself more comfortable when some of the data argued against this possibility. In any case, the wealth of cosmological data now available appears to unambiguously point to the fact that $\Omega_{\text {mat }}<1$, whether or not the cosmological constant is non-zero. This fact may have profound implications for dark matter detection.

\section{IMPLICATIONS FOR PARTICLE PHYSICS AND THE SEARCH FOR DARK MATTER}

The magnitude of the cosmological constant which would be required by the present data is remarkably small. Before proceeding to examine the consequences of the above results for dark matter detection, it is worth pausing for a moment to reflect on this feature. When I recently did this while preparing this lecture, I turned to a pocket calendar I had with me, and noticed the quotation:

"To see what is in front of one's nose requires a constant struggle"

George Orwell

What, you may ask, does this have to do with the topic at hand. Plenty, I claim. For it reminds us that we can put remarkably stringent limits on certain quantities by using macroscopic amounts of material. In particular, it harkens back to another famous quotation, this time from Maurice Goldhaber, who put one of the first limits on proton decay by declaring that if the proton had a lifetime less than about $10^{17}$ years, "You could feel it in your bones!". By this he meant that proton decays in our body would be so frequent that we would die from the radiation exposure.

In this spirit we can perform a similar experiment. Look at the end of your nose. Now, in a universe dominated by a cosmological constant, space begins to expand exponentially. One can calculate than for distances separated by larger than an amount $R>M_{P l} / 3 \Lambda^{1 / 2}$, points will have a relative velocity exceeding that of light, and thus will remain out of causal contact. Thus, the fact that you can see the end of your nose implies a bound $\Lambda<10^{-68} M_{P l}^{4}$ !

Of course, the fact that we can see distant galaxies gives us an even stronger bound. And, the fact that the cosmological constant affects dynamics on larger 
scales no more than it is claimed to by the present observations gives a bound $\Lambda<10^{-123} M_{P l}^{4}$. What makes this small number so hard to understand, in a cosmological context is not merely the "naturalness" problem of which particle physicists are aware, but rather, if this has been constant over cosmological time, this is the first time in the history of the universe when the energy density in a cosmological constant is comparable to the energy density of matter and radiation! It is for this reason that some cosmologists are driven to the idea that what is being observed is not really a cosmological constant, but something perhaps more exotic [16].

Be that as it may. Particle physicists will never measure a quantity of this magnitude directly in the laboratory. However, they may one day directly measure non-baryonic particles which presumably make up our galactic halo. And the new data brings good tidings in this regard. For the only well motivated candidates for Cold Dark Matter, axions and WIMPS one can write down a general relation:

$$
\sigma_{\text {detection }} \approx \frac{1}{\Omega_{D M}}
$$

The reasons for this are different for each candidate. For axions, one can understand the origin of this relation as follows: Axions are dark matter because at early times thier potential (considered as a function of an angular variable which can be taken to go from $-\pi$ to $\pi$ ) changes form:
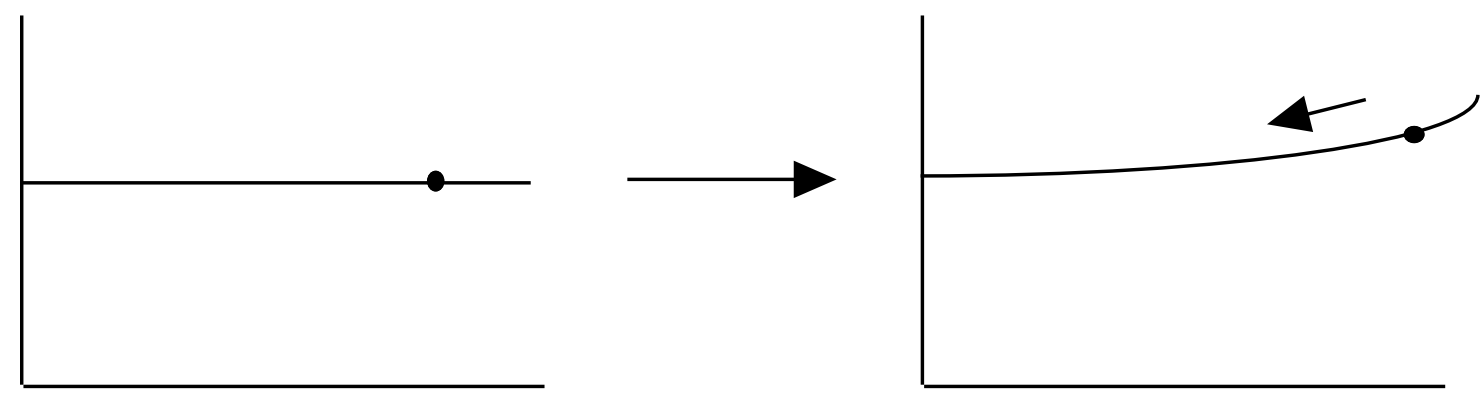

In the former case, no energy is stored in the axion field. However, once the axion gets a mass, energy is stored in the axion field, which then dynamically rolls to the bottom of its potential. However, the time it takes to begin rolling is inversely proportional to the curvature of its potential, and is thus inversely proportional to the axion mass. Thus, the smaller the axion mass, the longer the energy gets stored before it begins to redshift and the greater the remnant axion density. Since the axion couplings are inversely proportional to the axion mass, one therefore obtains the relation above.

For WIMPs, the situation is more direct. Remnant WIMPs results from incomplete annihilations in an initial thermal poulation, so that

$$
\Omega_{X} h^{2} \approx \frac{10^{-37} \mathrm{~cm}^{2}}{<\sigma_{a n n} v>}
$$


By crossing symmetry, the WIMP annihilation cross section is roughly proportional to the WIMP scattering cross section. Thus, as the WIMP abundance decreases, its scattering cross section generally increases.

Astute experimentalists may argue that this is a scam, because as the WIMP (axion) density decreases, the flux on Earth also decreases, so even if there are larger cross sections, the event rate will not change! However, this is wrong. Until the density decreases to the point (below about $\Omega_{x}<0.1$ ) when WIMPs (axions) do not have sufficient densities to account for all galactic halo dark matter, it is natural to assume that their galactic density is given by the halo density. Just because their overall cosmic density is insufficient to close the universe, this need not imply that their flux on earth is reduced!

\section{CONCLUSIONS}

It is time to throw in the towel and accept the paradigm shift in Cosmology. All evidence suggests that $\Omega_{D M}<1$. The dominant energy density in the universe may be far darker than that stored in dark matter - it may be stored in empty space itself! Nevertheless the news is good for direct detection of non-baryonic dark matter. Cross sections may be higher than previously invisaged when it was felt that Cold Dark Matter must result in a closure density all by itself.

Of course, as a theorist one tries to think beyond the next set of experiments. What if the next generation of WIMP detectors detects a signal, for example? What then? How will we be sure that it is from the galactic halo, and how can we learn about the halo properties, and/or the properties of the dark matter particles? I will close this lecture by advertising some new work we have been involved in which may shed some light on new WIMP signatures. First, by exploring the angular variation of the predicted WIMP signals which might arise from a variety of different models for our galactic halo, we have recently demonstrated [17] that as few as 15-20 events would be needed in a detector having directional sensitivity before a halo induced signal could be distinguished from a flat background of noise. Next, with T. Damour, I have recently demonstrated that there is likely to be a new solar system population of WIMPs existing in trapped Solar orbits intersecting the earth if the WIMP cross sections on matter are large enough to be detected at the next generation of detectors [18]. This population will produce a dramatically different signal in cryogenic detectors, and could be used as a discriminant to verify any previoulsy detected WIMP signal, or could be searched for independently.

I thank my collaborators involved in various aspects of the work described here, Michael Turner, Brian Chaboyer, Pierre Demarque, Peter Kernan, Craig Copi, Junseong Heo, and Thibault Damour. I also thank the those who organized a very stimulating and enjoyable meeting. 


\section{REFERENCES}

1. L.M. Krauss and M. S. Turner, J. Gen. Rel. Grav., 27,1137 (1995)

2. J.P. Ostriker and P. Steinhardt, Nature, 377, 600 (1995)

3. L.M. Krauss, Ap. J., 501, in press, (1998)

4. B. Chaboyer, P. Demarque, P. Kernan, and L. M. Krauss, Science, 271, 957 (1996)

5. W. L. Freedman, Proc. Nat. Acad. Sci., 95, 2 (1998)

6. B. Chaboyer, P. Demarque, P. Kernan, and L. M. Krauss, Ap. J. , 494, 96 (1998)

7. L. M. Krauss and P. J. Kernan, Phys. Lett. B347, 347 (1995)

8. C. Copi, D.N. Schramm, and M.S. Turner, Science, 267, 192 (1995)

9. D. Tytler, X. M. Fan, and S. Burles, Nature, 381, 207 (1996)

10. A. E. Evrard, MNRAS, in press (1998)

11. J.A. Peacock and S.J. Dodds, MNRAS, 267, 1020 (1994)

12. A. R. Liddle, D.H. Lyth, P.T.P. Viana, M. White, MNRAS, 282, 281 (1996)

13. N.A. Bahcall, X. Fan, Ap. J. 504, in press, (1998); Proc. Nat. Acad. Sci., 95, 5956 (1998)

14. S. Perlmutter et al, LBNL preprint 41801 (1998)

15. A. Reiss et al, preprint, submitted to Ap. J.

16. R. Caldwell et al, Phys. Rev. Lett., 80, 1582 (1998)

17. L.M. Krauss, Phys. Rep. (Proc. Workshop on Dark Matter Detection), in press; see also C. Copi, J. Heo and L.M.Krauss, in preparation.

18. T. Damour and L.M. Krauss, astro-ph/9806165 (1998) 